\title{
La responsabilidad social empresarial como estrategia de diferenciación de las empresas exportadoras
}

\section{Corporate social responsibility as a differentiation strategy for export companies}

Kelvin Ronaldo Rodriguez Eugenio

Universidad Técnica de Machala, Machala, Ecuador

krodrigue3@utmachala.edu.ec

(D) https://orcid.org/0000-0003-0389-5762

Jennifer Edith Gallo Apolo

Universidad Técnica de Machala, Machala, Ecuador

igallo2@utmachala.edu.ec

https://orcid.org/0000-0003-0870-2536

Mayiya Lisbeth González Illescas

Universidad Técnica de Machala, Machala, Ecuador

mlgonzalez@utmachala.edu.ec

(D) http://orcid.org/0000-0002-5219-3807

Luis Pastor Carmenate Fuentes

Universidad Técnica de Machala, Machala, Ecuador

lcarmenate@utmachala.edu.ec

http://orcid.org/0000-0001-6057-2746

Recepción: 21/07/2021 | Aceptación: 31/08/2021 | Publicación: 10/09/2021

Cómo citar (APA, séptima edición): Rodriguez Eugenio, K. R., Gallo Apolo, J. E., González Illescas, M. L., y Carmenate Fuentes, L. P. (2021). La responsabilidad social empresarial como estrategia de diferenciación de las empresas exportadoras. INNOVA Research Journal, 6(3), 171-189. https://doi.org/10.33890/innova.v6.n3.2021.1832

\section{Resumen}

Las empresas exportadoras se enfrentan a transformaciones constantes de los mercados y necesitan adoptar estrategias distintas a las tradicionales para diferenciarse de sus competidores. Ofrecer productos con precios accesibles y con altos estándares de calidad ya no son un diferencial, sino 
más bien, requisitos básicos. Actualmente, la responsabilidad social empresarial (RSE) se presenta como un diferencial para las empresas exportadoras que contribuyen a generar ventajas competitivas. En este contexto, el objetivo de esta investigación fue: caracterizar las prácticas o atributos de la RSE aplicadas en empresas exportadoras como estrategia de diferenciación. De forma complementaria se planteó la interrogante: ¿de qué manera esas estrategias de diferenciación basadas en la RSE contribuyen en la obtención de ventajas competitivas en las empresas exportadoras? Se empleó una investigación documental de enfoque cualitativo y alcance descriptivo con la aplicación de los métodos teóricos: inductivo-deductivo y analítico-sintético. Como resultado se tuvo que las empresas exportadoras realizan prácticas de RSE ligadas principalmente a las dimensiones social, económica y ambiental y a los estándares de las certificaciones ambientales o de responsabilidad social. Asimismo, se evidenciaron casos de empresas que destacan los atributos de sus productos mediante certificaciones orgánicas. Estas estrategias les han permitido la internacionalización e incursión en mercados exigentes, la creación de una imagen y reputación positiva y sobre todo la diferenciación ante sus competidores. Se concluye que las empresas exportadoras implementan estrategias de diferenciación mediante la aplicación de protocolos de RSE tanto a nivel producto como a nivel empresa, siendo esta última la de mayor uso.

Palabras claves: responsabilidad social empresarial; estrategia de diferenciación; empresas exportadoras; ventajas competitivas.

\begin{abstract}
Export companies face constant market transformations and need to adopt strategies other than traditional ones to differentiate themselves from their competitors. Offering products with affordable prices and high quality standards are no longer a differential, but rather basic requirements. Currently, corporate social responsibility (CSR) is presented as a differential for export companies that contribute to generating competitive advantages. In this context, the aim of this research was: to characterize CSR practices or attributes applied in export companies as a differentiation strategy. In addition, the question was raised: How do these CSR-based differentiation strategies contribute to obtaining competitive advantages in export companies? A documentary research of qualitative approach and descriptive scope was used with the application of theoretical methods: inductive-deductive and analytical-synthetic. As a result, export companies perform CSR practices mainly linked to social, economic and environmental dimensions and standards of environmental or social responsibility certifications. There were also cases of companies that highlight the attributes of their products through organic certifications. These strategies have enabled them to internationalize and incur demanding markets, create a positive image and reputation, and above all the differentiation to their competitors. It's concluded that export companies implement differentiation strategies by applying CSR protocols at both product and enterprise level, the latter being the most widely used.

Keywords: corporate social responsibility; differentiation strategy; export companies; competitive advantages.
\end{abstract}




\section{Introducción}

Las empresas exportadoras deben gestionar un elevado nivel de competitividad frente a sus similares. Precios accesibles y el cumplimiento de estrictos estándares de calidad ya no son un diferencial, sino más bien requisitos básicos para acceder y permanecer en los mercados internacionales (Marakova, Wolak y Tuckova, 2021). Las empresas necesitan adoptar estrategias distintas a las tradicionales para diferenciarse de sus competidores (Martos, Cortés y Jiménez, 2019).

La Responsabilidad Social Empresarial (en adelante denominada RSE) surge como un referente de estrategia enfocada en cubrir las necesidades y expectativas de un creciente grupo de consumidores, distribuidores y minoristas que demandan un mayor compromiso social y ambiental de las empresas globales a quienes adquieren sus productos. Un importante grupo de investigadores ha puesto su atención en analizar la RSE y su relación con las empresas que operan en mercados internacionales. Así, se destacan las aportaciones de Tan, Ho, Pidani y Goveravaram (2021) en las cuales se destaca la RSE como una inversión estratégica. Existe consenso de criterios al considerar a la RSE como una herramienta estratégica (Barić y Omazić, 2018; Flores, Vega y Chávez, 2016; Marakova et al. 2021; Nyuur, Ofori y Amponsah, 2019; Rueda, 2017), o como una fuente para generar oportunidades en segmentos de mercado (Boubakary y Moskolaï, 2016; Porter y Kramer, 2006).

En el contexto de las empresas exportadoras, la RSE puede verse como una estrategia empresarial que asegura el éxito y la competitividad en mercados internacionales (Godos, Cabeza y Fernández, 2018; Parrales, Trelles y González, 2021). Así, en países desarrollados donde existe una población que refleja preocupación por los problemas sociales y ambientales, los consumidores se muestran más dispuestos a pagar un sobreprecio por productos de empresas socialmente responsables (Boehe y Barin, 2010).

En función de lo expuesto, este artículo tiene por objetivo: caracterizar las prácticas o atributos de la RSE aplicadas en empresas exportadoras como estrategia de diferenciación. De forma complementaria se plantea una interrogante como guía de la investigación: ¿de qué manera esas estrategias de diferenciación basadas en la RSE contribuyen en la obtención de ventajas competitivas en las empresas exportadoras?

Para dar respuesta a dicha interrogante y por ende cumplir con el objetivo propuesto, se lleva a cabo un estudio de tipo documental, con enfoque cualitativo y alcance descriptivo, con el apoyo de publicaciones científicas previas que cumplen criterios asociados al objeto de estudio. Los métodos científicos que se emplean son el analítico-sintético e inductivo-deductivo, tanto en el proceso de búsqueda y selección de referencias bibliográficas, como en el proceso de redacción del artículo.

La estructura del documento se presenta de la siguiente manera. Posterior a la introducción, se contempla la revisión de literatura que aborda nociones teóricas de la RSE. El apartado de metodología recoge la explicación de los métodos, procedimientos y recursos empleados. Se presentan los resultados mediante la sistematización de 12 estudios previos y su correspondiente 
análisis. Finalmente, se contemplan las conclusiones, posibles limitaciones del estudio y temas que se podrían considerar para abordar en futuras investigaciones.

\section{Marco teórico}

\section{Nociones teóricas sobre la RSE}

Para una aproximación a la RSE, a continuación, se revisa el significado de cada una de sus palabras componentes. El término responsabilidad hace alusión al compromiso u obligación que tienen las empresas ante los impactos que sus acciones puedan generar en la sociedad. Por su parte, el término social hace referencia a la sociedad, que engloba además de los stakeholders (grupos de interés) a los animales, plantas y medio ambiente que puedan resultar afectados por las acciones de una empresa. Y el término empresarial o corporativo incluye a toda empresa o negocio independientemente de su forma jurídica o tamaño (Carroll y Brown, 2018).

Se atribuye a Howard Bowen la primera definición de RSE, en la década de los 50, con su publicación titulada: Social Responsibilities of the Businessmen (Carroll, 1979; Chavarría, Ganga y García, 2020; Daniel, Alvarado, Sansores y Navarrete, 2019; Lizcano y Lombana, 2018; Morán, Cárdenas y Córdova, 2019; Ormaza, Ochoa, Ramírez y Quevedo, 2020).

Resulta complejo llegar a una definición precisa y universal sobre la RSE a pesar de que ha sido un tema ampliamente abordado (Bogetić, Đorđević, Ćoćkalo y Vorkapić, 2018; Castejón y López, 2016; Kolk, 2016; Morán et al. 2019; Suárez y Yance, 2020) por lo que no existe una forma precisa de describirla y aplicarla en el campo empresarial (Martos et al. 2019; Villafán, 2020). En esta línea de criterios, Cueto y de la Cuesta (2019) destacan que "Dependiendo de los agentes involucrados, empresas, sindicatos, administración y sociedad civil, la interpretación del término RSE es diferente" (p. 43).

El estudio de la RSE se ha desarrollado desde diversas teorías y enfoques (Garriga y Melé, 2004; Lizcano y Lombana, 2018; Martos et al. 2019; Mateos, Estrada y Hernández, 2020). A continuación, en la tabla 1 se expone a manera de sistematización una explicación relativa a cuatro teorías sobre la RSE, a saber: instrumentales, políticas, integradoras y éticas.

\section{Tabla 1}

Clasificación de teorías y enfoques sobre la RSE

\begin{tabular}{clcl}
\hline \multicolumn{1}{c}{ Teorías } & \multicolumn{2}{c}{ Descripción } & Enfoques \\
\hline Instrumentales & $\begin{array}{l}\text { Las empresas aplican la RSE como } \\
\text { herramienta estratégica para la consecución de } \\
\text { resultados económicos y creación de riqueza. }\end{array}$ & $\begin{array}{l}\text { Estrategias para lograr ventaja competitiva; y } \\
\text { Marketing relacionado a la causa. }\end{array}$
\end{tabular}




\begin{tabular}{llll}
\hline \multicolumn{1}{c}{ Teorías } & \multicolumn{1}{c}{ Descripción } & \multicolumn{1}{c}{ Enfoques } \\
\hline Políticas & $\begin{array}{l}\text { Las empresas aplican la RSE en sus } \\
\text { interacciones con la sociedad para proteger su } \\
\text { poder social y posición. }\end{array}$ & $\begin{array}{l}\text { Constitucionalismo corporativo; } \\
\text { Integradora del contrato social; y Ciudadanía } \\
\text { corporativa. }\end{array}$ \\
Integradoras & $\begin{array}{l}\text { Las empresas buscan lograr su legitimidad, } \\
\text { aceptación y permanencia en el mercado } \\
\text { tomando en cuenta las demandas sociales. }\end{array}$ & $\begin{array}{l}\text { Gestión de problemas; El principio de } \\
\text { responsabilidad pública; Gestión de las partes } \\
\text { interesadas; y Desempeño social corporativo. }\end{array}$ \\
& $\begin{array}{l}\text { Las empresas basan su relación con la } \\
\text { sociedad con comportamientos éticos y la } \\
\text { asumen como una obligación ética. }\end{array}$ & $\begin{array}{l}\text { Teoría normativa de las partes interesadas; } \\
\text { Derechos universales; Desarrollo sostenible; y } \\
\text { Enfoque del bien común. }\end{array}$ \\
\hline
\end{tabular}

Fuente: Elaboración propia a partir de Garriga y Melé (2004).

Para exponer la evolución de las teorías de RSE, en la tabla 2 se resumen las principales publicaciones realizadas en torno a la RSE con base en la información obtenida en Carroll (2008), Garriga y Melé (2004) y Latapí, Jóhannsdóttir y Davídsdóttir (2019). Se identifica que la teoría más reciente que queda fuera de la visión de los autores descritos es la de creación de valor compartido de Porter y Kramer (2011), Trapp (2012) y Chandler (2016) por lo que se la ha clasificado dentro de la teoría instrumental, exactamente como estrategia para lograr ventajas competitivas.

\section{Tabla 2}

Enfoques de RSE según su evolución conceptual

\begin{tabular}{llll}
\hline \multicolumn{1}{c}{ Décadas } & \multicolumn{1}{c}{ Publicaciones } & \multicolumn{1}{c}{ Enfoque / Teoría } \\
\hline $\begin{array}{l}\text { Década de } \\
1950\end{array}$ & $\begin{array}{l}\text { Social Responsibilities of the Businessman de Howard Bowen } \\
\text { (1953) }\end{array}$ & Primera definición formal de la RSE \\
\hline $\begin{array}{l}\text { Década de } \\
1960\end{array}$ & $\begin{array}{l}\text { Can Business Afford to Ignore Corporate Social } \\
\text { Responsibilities? de Keith Davis (1960) }\end{array}$ & $\begin{array}{l}\text { Constitucionalismo corporativo / } \\
\text { Teoría política }\end{array}$ \\
\hline $\begin{array}{l}\text { Década de } \\
1970\end{array}$ & $\begin{array}{l}\text { The Social Responsibility of Business is to Increase its Profits } \\
\text { de Milton Friedman (1970) }\end{array}$ & $\begin{array}{l}\text { Maximización del valor para el } \\
\text { accionista/ Teoría instrumental }\end{array}$ \\
\cline { 2 - 5 } & $\begin{array}{l}\text { The Case for and against Business Assumption of Social } \\
\text { Responsibilities de Keith Davis (1973) }\end{array}$ & $\begin{array}{l}\text { Ciudadanía corporativa / Teoría } \\
\text { política }\end{array}$ \\
\cline { 2 - 5 } & $\begin{array}{l}\text { Dimensions of Corporate Social Performance: An Analytical } \\
\text { Framework de Sethi (1975) }\end{array}$ & $\begin{array}{l}\text { Gestión de problemas / Teoría } \\
\text { integradora }\end{array}$ \\
\cline { 2 - 5 } & $\begin{array}{l}\text { Private Management and Public Policy. The Principle of } \\
\text { Public Responsibility de Lee Preston y James Post (1975) }\end{array}$ & $\begin{array}{l}\text { Principio de responsabilidad } \\
\text { púba / Teoría integradora }\end{array}$ \\
\cline { 2 - 5 } & $\begin{array}{l}\text { A Three-Dimensional Conceptual Model of Corporate } \\
\text { Performance de Archie Carroll (1979) }\end{array}$ & $\begin{array}{l}\text { Desempeño social corporativo / } \\
\text { Teoría integradora }\end{array}$ \\
\hline
\end{tabular}

Esta obra se comparte bajo la licencia Creative Common Atribución-No Comercial 4.0 International (CC BY-NC 4.0) Revista de la Universidad Internacional del Ecuador. URL: https://www.uide.edu.ec/ 
Kelvin Ronaldo Rodriguez Eugenio, Jennifer Edith Gallo Apolo, Mayiya Lisbeth González Illescas y Luis Pastor Carmenate Fuente

ISSN 2477-9024. Innova Research Journal (Septiembre-Diciembre, 2021). Vol. 6, No. 3, pp. 171-189

\begin{tabular}{|c|c|c|}
\hline Décadas & Publicaciones & Enfoque / Teoría \\
\hline \multirow[t]{3}{*}{$\begin{array}{l}\text { Década de } \\
1980\end{array}$} & $\begin{array}{l}\text { Corporate Social Responsibility Revisited, Redefined de } \\
\text { Thomas Jones (1980) }\end{array}$ & $\begin{array}{l}\text { Gestión de problemas / Teoría } \\
\text { integradora }\end{array}$ \\
\hline & $\begin{array}{l}\text { Strategic Management: A Stakeholder Approach de Edward } \\
\text { Freeman (1984) }\end{array}$ & $\begin{array}{l}\text { Teoría normativa de las partes } \\
\text { interesadas / Teoría ética }\end{array}$ \\
\hline & $\begin{array}{l}\text { The Evolution of the Corporate Social Performance Model de } \\
\text { Steven Wartick \& Philip Cochran (1985) }\end{array}$ & $\begin{array}{l}\text { Desempeño social corporativo / } \\
\text { Teoría integradora }\end{array}$ \\
\hline \multirow[t]{6}{*}{$\begin{array}{l}\text { Década de } \\
1990\end{array}$} & $\begin{array}{l}\text { Corporate Social Performance Revisited de Donna Wood } \\
\text { (1991) }\end{array}$ & $\begin{array}{l}\text { Desempeño social corporativo / } \\
\text { Teoría integradora }\end{array}$ \\
\hline & $\begin{array}{l}\text { The Politics of Stakeholder Theory: Some Future Directions } \\
\text { de Edward Freeman (1994) }\end{array}$ & $\begin{array}{l}\text { Teoría normativa de las partes } \\
\text { interesadas / Teoría ética }\end{array}$ \\
\hline & $\begin{array}{l}\text { Addressing a Theoretical Problem by Reorienting the } \\
\text { Corporate Social Performance Model de Diane Swanson } \\
\text { (1995) }\end{array}$ & $\begin{array}{l}\text { Desempeño social corporativo / } \\
\text { Teoría integradora }\end{array}$ \\
\hline & $\begin{array}{l}\text { The Stakeholder Theory of the Corporation: Concepts, } \\
\text { Evidence, and Implications de Thomas Donaldson y Lee } \\
\text { Preston (1995) }\end{array}$ & $\begin{array}{l}\text { Teoría normativa de las partes } \\
\text { interesadas / Teoría ética }\end{array}$ \\
\hline & $\begin{array}{l}\text { How Corporate Social Responsibility pays off de Lee Burke \& } \\
\text { Jeanne Logsdon (1996) }\end{array}$ & $\begin{array}{l}\text { Estrategias para lograr ventaja } \\
\text { competitiva/Teoría instrumental }\end{array}$ \\
\hline & $\begin{array}{l}\text { Corporate Social Responsibility: Evolution of Definitional } \\
\text { Construct de Archie Carroll (1999) }\end{array}$ & $\begin{array}{l}\text { Ciudadanía corporativa / Teoría } \\
\text { política }\end{array}$ \\
\hline \multirow[t]{7}{*}{$\begin{array}{l}\text { Década de } \\
2000\end{array}$} & $\begin{array}{l}\text { Objetivos de Desarrollo del Milenio (ODM) de la Naciones } \\
\text { Unidas (2000) }\end{array}$ & Derechos universales / Teoría ética \\
\hline & $\begin{array}{l}\text { Stakeholder Theory: A Libertarian Defense de Edward } \\
\text { Freeman y Robert Phillips (2002) }\end{array}$ & $\begin{array}{l}\text { Teoría normativa de las partes } \\
\text { interesadas / Teoría ética }\end{array}$ \\
\hline & $\begin{array}{l}\text { The Competitive Advantage of Corporate Philanthropy de } \\
\text { Michael Porter \& Mark Kramer (2002) }\end{array}$ & $\begin{array}{l}\text { Estrategias para lograr ventaja } \\
\text { competitiva /Teoría instrumental }\end{array}$ \\
\hline & $\begin{array}{l}\text { Concepts and Definitions of CSR and Corporate } \\
\text { Sustainability: Between Agency and Communion de Marcel } \\
\text { Van Marrewijk (2003) }\end{array}$ & Desarrollo sostenible / Teoría ética \\
\hline & $\begin{array}{l}\text { Corporate Social Responsibility: A Three-Domain Approach } \\
\text { de Mark Schwartz y Archie Carroll (2003) }\end{array}$ & $\begin{array}{l}\text { Desempeño social corporativo / } \\
\text { Teoría integradora }\end{array}$ \\
\hline & $\begin{array}{l}\text { Strategy \& Society: The link between competitive advantage } \\
\text { and corporate social responsibility de Michael Porter \& Mark } \\
\text { Kramer (2006) }\end{array}$ & $\begin{array}{l}\text { Estrategias para lograr ventaja } \\
\text { competitiva /Teoría instrumental }\end{array}$ \\
\hline & Guidance on social responsibility ISO 26000 (2010) & Desarrollo sostenible / Teoría ética \\
\hline
\end{tabular}




\begin{tabular}{|c|c|c|}
\hline Décadas & Publicaciones & Enfoque / Teoría \\
\hline \multirow{5}{*}{$\begin{array}{l}\text { Década de } \\
2010\end{array}$} & $\begin{array}{l}\text { Creating shared value de Michael Porter \& Mark Kramer } \\
\text { (2011) }\end{array}$ & $\begin{array}{l}\text { Estrategias para lograr ventaja } \\
\text { competitiva/Teoría instrumental }\end{array}$ \\
\hline & $\begin{array}{l}\text { Estrategia renovada de la UE para } 2011-2014 \text { sobre la } \\
\text { responsabilidad social de la Comisión Europea (2011) }\end{array}$ & Desarrollo sostenible / Teoría ética \\
\hline & $\begin{array}{l}\text { Corporation as climate ambassador: Transcending business } \\
\text { sector boundaries in a Swedish CSR campaign de Leila Trapp } \\
(2012)\end{array}$ & $\begin{array}{l}\text { Estrategias para lograr ventaja } \\
\text { competitiva/Teoría instrumental }\end{array}$ \\
\hline & $\begin{array}{l}\text { Objetivos de Desarrollo Sostenible (ODS) de la Naciones } \\
\text { Unidas (2015) }\end{array}$ & Desarrollo sostenible / Teoría ética \\
\hline & $\begin{array}{l}\text { Strategic corporate social responsibility: sustainable value } \\
\text { creation de David Chandler (2016) }\end{array}$ & $\begin{array}{l}\text { Estrategias para lograr ventaja } \\
\text { competitiva/Teoría instrumental }\end{array}$ \\
\hline
\end{tabular}

Fuente: Elaboración propia a partir de Carroll (2008); Garriga y Melé (2004); y Latapí et al. (2019).

Como se puede constatar, la primera definición formal sobre la RSE surgió en 1953 con Bowen. Posteriormente, en la década de 1960 Davis concibió la RSE desde la relación empresasociedad. En la década de 1970 Friedman rechaza la RSE mencionando que la maximización del valor para el accionista era la única responsabilidad social de las empresas. Para finales de la década de 1970, Carroll contribuyó con un modelo conceptual de desempeño social corporativo. Las décadas de 1980 y 1990 se caracterizaron por contener temas complementarios a la RSE, tales como desempeño social corporativo, teoría normativa de las partes interesadas y ciudadanía corporativa. Finalmente, desde el año 2000 hasta la actualidad, varios autores coinciden en resaltar las contribuciones de las RSE al desarrollo sostenible y su posicionamiento como instrumento de gestión para la obtención de ventajas competitivas.

De acuerdo al objetivo de este estudio, se asume la responsabilidad social empresarial desde la teoría instrumental, específicamente bajo el enfoque estratégico desde el cual se reconoce su potencial para generar ventajas competitivas (Boubakary y Moskolaï, 2016; Porter y Kramer, 2006; Tan et al. 2021).

Siguiendo la teoría de Porter (1985) la ventaja competitiva es aquel valor superior que una empresa crea para sus clientes mediante estrategias genéricas: 1) liderazgo en costos, desde el cual la empresa busca ser competitiva mediante precios más bajos que sus rivales; 2) diferenciación, que se aplica cuando la empresa busca distanciarse de sus competidores mediante atributos únicos que son altamente valorados y recompensados por sus clientes; y 3) enfoque, que se la puede considerar como complementaria a las estrategias anteriores ya que la empresa puede aplicar la estrategia de liderazgo en costo o la de diferenciación en un segmento objetivo.

En este sentido, la RSE encaja dentro de la estrategia de diferenciación ya que en mercados donde existen preocupaciones por cuestiones sociales y éticas, las empresas pueden incorporar atributos de RSE en sus productos o servicios y gozar así de una ventaja competitiva (Fong, Parra, Soriano y Teodoro, 2020). Así, la certificación de RSE puede aumentar las posibilidades de que 
los consumidores tiendan a preferir bienes de empresas socialmente responsables y estarán dispuestos a reconocerlas pagando precios superiores (Nazzaro, Stanco y Marotta, 2020). Por ende, las empresas socialmente responsables tendrán una posición superior en el mercado frente a aquellas que no aplican los principios de la RSE.

Aunado a lo anterior, se puede decir que incorporar la RSE en la estrategia puede ser una fuente de oportunidades para las empresas (Boubakary y Moskolaï, 2016) ya que permite crear una buena imagen, una reputación positiva, un mejor posicionamiento y por tanto la obtención de ventajas competitivas (Castro, 2017; Boubakary y Moskolaï, 2016; Lu, Ren, Yao, Qiao, Mikalauskiene y Streimikis, 2020; Nazzaro et al. 2020; Vu, Nguyen, Nguyen, Nguyen y Nguyen, 2020). Pero para que esto sea posible, es necesario que los consumidores conozcan los atributos del producto y/o las prácticas de RSE implementadas (Mateos et al. 2020) por lo que la comunicación es de vital importancia, siendo las principales herramientas de comunicación las redes sociales, los informes anuales de RSE, las páginas web y eventos de promoción (Kim, 2019).

\section{Metodología}

La investigación es de tipo documental, con enfoque cualitativo y alcance descriptivo. Los materiales que se han empleado corresponden a publicaciones científicas previas, cuya selección responde a dos estrategias, una relacionada a la identificación de artículos en bases de datos y otra al análisis de las referencias bibliográficas de los artículos obtenidos de la estrategia anterior.

Las bases de datos consultadas fueron Researchgate, Redib, Scopus, ProQuest, Science Direct y Taylor \& Francis, además del motor de búsqueda Google Académico. Las búsquedas se realizaron por título, resumen y palabras claves, utilizando las siguientes palabras de búsqueda "evolución conceptual de la RSE", "enfoques y teorías de RSE", "enfoque estratégico de RSE para la obtención de ventajas competitivas", "responsabilidad social empresarial como estrategia de diferenciación", "responsabilidad social empresarial como fuente de ventaja competitiva", "responsabilidad social empresarial como estrategia para competir en mercados internacionales", "responsabilidad social empresarial en empresas exportadoras" en el idioma español, inglés y portugués.

En esta etapa fue fundamental la lectura superficial de la introducción de los artículos encontrados y aquellos que fueron preseleccionados se organizaron en una ficha bibliográfica de contenido. De estos artículos preseleccionados se hizo una revisión de las referencias bibliográficas (segunda estrategia de búsqueda). Posteriormente, la lectura de reconocimiento y el método de análisis permitieron hacer la depuración de las referencias bibliográficas obtenidas, es decir, se seleccionaron aquellos que cumplían con los siguientes criterios de inclusión:

- Las fuentes de información deberán ser artículos científicos, artículos de revisión, libros y capítulos de libros.

- Las publicaciones deberán estar comprendidas entre los años 2016 y 2021 a excepción de autores clásicos. 
- Para el apartado de resultados, las publicaciones deberán describir prácticas o atributos de RSE como estrategia de diferenciación para la obtención de ventajas competitivas, desarrolladas específicamente por empresas exportadoras (ya sea de manera teórica o empírica).

Tanto en la revisión de literatura como en resultados y discusión se implementaron los métodos teóricos analítico-sintético e inductivo-deductivo. El análisis se implementa al extraer información relevante (lectura de reconocimiento) y la síntesis al ordenar y relacionar dicha información extraída (redacción). El método inductivo-deductivo se aplicó en la revisión de literatura precisamente para identificar los enfoques y teorías de RSE de los diversos estudios publicados a lo largo de las décadas.

\section{Resultados y Discusión}

Para Barin, Boehe y Ogasavara (2015) las empresas exportadoras pueden implementar estrategias de diferenciación basadas en la RSE tanto a nivel producto (atributos) como a nivel empresa (prácticas o acciones). En ese sentido, se considera estrategia de diferenciación a nivel producto cuando éste posee una certificación social o ambiental, cuando es producido usando materias primas con certificación social o ambiental, o cuando el producto es reconocido como social o ambientalmente responsable. Por otro lado, se considera estrategia de diferenciación a nivel empresa cuando se desarrollan proyectos en beneficio de la comunidad en la que opera, $o$ cuando la empresa es reconocida a nivel local o internacional por implementar prácticas social o ambientalmente responsables.

A la luz de lo anterior, se identificaron 12 estudios previos que describen prácticas de RSE aplicadas por empresas exportadoras. Los resultados fueron sistematizados en la tabla 3 que contiene información relacionada al autor y año de publicación, muestra (empresa exportadora, sector y país), prácticas o atributos de RSE (estrategia de diferenciación) y a las ventajas competitivas obtenidas.

\section{Tabla 3}

Casos de empresas exportadoras que aplican RSE como estrategia de diferenciación

\begin{tabular}{|c|c|c|c|}
\hline Autor & Muestra & Prácticas o atributos de RSE & Ventaja competitiva obtenida \\
\hline $\begin{array}{l}\text { Granados, } \\
\text { Arredondo } \\
\text { y Caldera } \\
(2017)\end{array}$ & $\begin{array}{lr}10 & \text { pymes } \\
\text { exportadoras } & \text { del } \\
\text { Estado de Guanajuato } \\
\text { México. }\end{array}$ & $\begin{array}{l}\text { Con una medición de la RSE } \\
\text { mediante las dimensiones de la ISO } \\
26000 \text { los resultados demostraron } \\
\text { que las pymes realizan prácticas de } \\
\text { RSE principalmente relacionadas a } \\
\text { las dimensiones: Participación } \\
\text { Activa y Desarrollo de la } \\
\text { Comunidad; Medio Ambiente; y } \\
\text { Derechos Humanos. }\end{array}$ & $\begin{array}{l}\text { Se evidenció que las pymes ven a la } \\
\text { RSE como una forma de crecimiento } \\
\text { y/o internacionalización ya que les ha } \\
\text { permitido exportar a mercados } \\
\text { exigentes a pesar de no ser grandes } \\
\text { empresas o reconocidas en México. } \\
\text { Siendo los principales destinos de } \\
\text { exportación Estados Unidos, Canadá } \\
\text { y países europeos. }\end{array}$ \\
\hline
\end{tabular}


Kelvin Ronaldo Rodriguez Eugenio, Jennifer Edith Gallo Apolo, Mayiya Lisbeth González Illescas y Luis Pastor Carmenate Fuente

ISSN 2477-9024. Innova Research Journal (Septiembre-Diciembre, 2021). Vol. 6, No. 3, pp. 171-189

\begin{tabular}{|c|c|c|c|}
\hline Autor & Muestra & Prácticas o atributos de RSE & Ventaja competitiva obtenida \\
\hline $\begin{array}{l}\text { Santa y } \\
\text { Fajardo } \\
(2017)\end{array}$ & $\begin{array}{l}3 \text { pymes exportadoras } \\
\text { del Departamento del } \\
\text { Quindío Colombia, a } \\
\text { saber: 1) } \\
\text { Quindío, Café } \\
\text { Promadeco y } \\
\text { Plásticos Fénix Ltda. }\end{array}$ & $\begin{array}{l}\text { 1) Café Quindío realiza prácticas } \\
\text { sociales y medioambientales } \\
\text { certificadas por Rainforest } \\
\text { Alliance, ISO 9001 y UTZ } \\
\text { Certified-Good Inside. Además, su } \\
\text { café cuenta con la certificación } \\
\text { USDA Organic. 2) Promadeco } \\
\text { realiza prácticas sociales y } \\
\text { medioambientales tales como: uso } \\
\text { responsable de la materia prima } \\
\text { (madera), apoyo a la reforestación } \\
\text { rápida de bosques, entre otras. 3) } \\
\text { Plásticos Fénix Ltda., realiza } \\
\text { prácticas sociales } \\
\text { medioambientales tales como: } \\
\text { contribución al desarrollo de la } \\
\text { comunidad y la preservación del } \\
\text { medio ambiente, entre otras. }\end{array}$ & $\begin{array}{l}\text { 1) Café Quindío ha logrado exportar } \\
\text { y consolidar su producto estrella } \\
\text { (café) a países como Rusia, España y } \\
\text { Chile gracias a sus prácticas de RSE } \\
\text { y sus certificaciones principalmente } \\
\text { la USDA Organic. 2) Promadeco ha } \\
\text { logrado incursionar mediante } \\
\text { exportaciones de muebles y } \\
\text { productos de carpintería en mercados } \\
\text { como Aruba, Panamá y Estados } \\
\text { Unidos. 3) Plásticos Fénix Ltda., sus } \\
\text { prácticas de RSE les ha permitido la } \\
\text { internacionalización ar mediante } \\
\text { exportaciones al mercado } \\
\text { ecuatoriano. }\end{array}$ \\
\hline
\end{tabular}

Colovic y 4 pymes fabricantes 1) En Cheese Co., la RSE es una Henneron de alimentos de parte integral de la empresa por lo (2018) Francia con que fomenta el respeto a las operaciones en personas, al ganado, el ecosistema y mercados extranjeros: al medio ambiente. 2) En Chocolate 1) Cheese Co., 2) Co., no se percibe la RSE como Chocolate Co., 3) parte de su estrategia, sin embargo, Wine Co., y 4) realiza ciertas actividades de RSE Smoothies Co. relacionadas a lo económico, medioambiental y social. 3) En Wine Co., se promueve la RSE como parte de su estrategia comercial basándose en el respeto al medio ambiente y el comercio ético. 4) En Smoothies Co., la RSE forma parte de su estrategia y se enfoca en el respeto al personal y el respeto al medio ambiente.

\begin{tabular}{|c|c|c|}
\hline \multirow{9}{*}{$\begin{array}{l}\text { Martos et } \\
\text { al. (2019) }\end{array}$} & empresas & Los resultados demostraron que las \\
\hline & $\begin{array}{l}\text { exportadoras del } \\
\text { sector }\end{array}$ & $\begin{array}{l}\text { empresas objeto de } \begin{array}{r}\text { estudio } \\
\text { implementan }\end{array}\end{array}$ \\
\hline & agroalimentario & agroecológicas que les han \\
\hline & $\begin{array}{l}\text { Almería, sureste de } \\
\text { España. }\end{array}$ & $\begin{array}{l}\text { permitido usar eficientemente sus } \\
\text { recursos para tratar así de disminuir }\end{array}$ \\
\hline & & sus impactos medioambientales. \\
\hline & & Además las empresas realizan \\
\hline & & prácticas y acciones de RSE \\
\hline & & enfocadas a los empleados, socios, \\
\hline & & $\begin{array}{l}\text { clientes, agricultores, comunidad y } \\
\text { competidores. }\end{array}$ \\
\hline
\end{tabular}

1) Para Cheese Co., sus prácticas de RSE les permitió crear una imagen positiva de la empresa en mercados exteriores. 2) Smoothies Co., se benefició de sus actividades de RSE obteniendo ventaja competitiva a nivel internacional. 3) Para Wine Co., la RSE le ha facilitado la incursión en mercados europeos con altas expectativas de RSE, donde los consumidores pueden pagar un sobreprecio por un vino responsable y ético. 4) En el caso de Chocolate Co., que fue la empresa con menos nivel de aplicación de RSE, ha optado por exportar a algunos países asiáticos donde las exigencias de adoptar prácticas de RSE es baja.

Se demostró que el compromiso asumido en materia de RSE impacta positivamente en las variables tangibles (desempeño financiero; desempeño exportador) e intangibles (imagen y reputación; y satisfacción de stakeholders). Además la RSE puede ayudar a las empresas a desarrollar una estrategia de diferenciación basada en las características sociales y ambientales de sus productos. 


\begin{tabular}{|c|c|c|c|}
\hline Autor & Muestra & Prácticas o atributos de RSE & Ventaja competitiva obtenida \\
\hline $\begin{array}{l}\text { Moncada, } \\
\text { Ramírez y } \\
\text { González } \\
(2019)\end{array}$ & $\begin{array}{l}5 \text { empresas } \\
\text { camaroneras } \\
\text { exportadoras de } \\
\text { Ecuador. }\end{array}$ & $\begin{array}{l}\text { Los resultados demostraron que las } \\
\text { empresas objeto de estudio } \\
\text { implementan estrategias de } \\
\text { diferenciación basadas en la } \\
\text { sustentabilidad, compromiso social } \\
\text { y calidad-trazabilidad, es por ello } \\
\text { que cuentan con certificaciones } \\
\text { internacionales tales como ASC, } \\
\text { BAP, Global G.A.P., AB, } \\
\text { Naturland, Sedex, Punto verde, } \\
\text { BRC, HACCP y BASC. }\end{array}$ & $\begin{array}{l}\text { Las certificaciones de gestión de la } \\
\text { calidad, de buenas prácticas de } \\
\text { manufactura, de sustentabilidad y de } \\
\text { responsabilidad social les han } \\
\text { permitido a las empresas cumplir con } \\
\text { las normativas, los estándares de } \\
\text { calidad y las exigencias de los } \\
\text { mercados internacionales, además de } \\
\text { generar confianza a sus } \\
\text { consumidores y sobre todo } \\
\text { diferenciarse de sus competidores. }\end{array}$ \\
\hline
\end{tabular}

$\begin{array}{lll}\begin{array}{l}\text { Mora, } \\ \text { Lituma }\end{array} \text { y } & \begin{array}{l}\text { exportadoras del } \\ \text { sector cacao, banano, }\end{array} & \text { Las empresas objeto de estudio } \\ \text { González } & \text { camarón, café y flores } & \text { procesos productivos mediante } \\ (2019) & \text { de Ecuador. } & \text { certificaciones ambientales, de } \\ & & \text { calidad, de seguridad alimentaria, } \\ & \text { de producción orgánica y de } \\ & \text { responsabilidad social. }\end{array}$

Los resultados demostraron que estas empresas han adoptado certificaciones para generar confianza al consumidor, beneficiándose así de una mayor acogida en los distintos mercados objetivos (internacionales) además que les ha permitido diferenciarse de otras empresas de productos similares.

Morán et Exportadora de Para lograr sus objetivos de RSE, la al. (2019) banano "Hacienda hacienda cuenta con la certificación Nueva Colonia" de de sostenibilidad Rainforest Ecuador.
Alliance que garantiza el cumplimiento de acciones relacionadas a lo social, económico y ambiental. En ese sentido, la Hacienda Nueva Colonia realiza programas de RSE en beneficio de sus trabajadores, comunidad y medio ambiente.
La certificación Rainforest Alliance le ha dado un valor agregado al producto permitiéndole a la Hacienda Nueva Colonia ingresar a mercados exigentes en cuanto a la verificación de productos. Asimismo la implementación de programas de RSE le ha permitido tener una buena relación con la comunidad, colaboradores y medio ambiente.

Estadísticamente se demostró que la RSE impacta positiva y significativamente en la ventaja competitiva de las empresas. Asimismo los resultados de las entrevistas demostraron que la RSE ayuda a atraer nuevos clientes, mejorar la relación con la comunidad, crear una buena imagen y reputación, que de alguna u otra manera se convierten en ventajas competitivas.

\begin{tabular}{lllll}
\hline Mejía y & Cementos Argos S.A & Esta empresa realiza acciones de & Mediante sus estrategias de \\
Bom & de Colombia. & RSE ligadas a los Objetivos de & sostenibilidad basadas en los ODS, \\
$(2020)$ & & Desarrollo Sostenible (ODS) Cementos Argos S.A. ha podido \\
& específicamente: Trabajo decente y diferenciarse de sus competidores y \\
& crecimiento económico (ODS 8); obtener ventajas competitivas que
\end{tabular}


Kelvin Ronaldo Rodriguez Eugenio, Jennifer Edith Gallo Apolo, Mayiya Lisbeth González Illescas y Luis Pastor Carmenate Fuente

ISSN 2477-9024. Innova Research Journal (Septiembre-Diciembre, 2021). Vol. 6, No. 3, pp. 171-189

\begin{tabular}{llll}
\hline Autor & Muestra & Prácticas o atributos de RSE & \multicolumn{2}{c}{ Ventaja competitiva obtenida } \\
\hline & La industria, la innovación e & trascienden las fronteras, es decir, \\
infraestructura (ODS 9); Ciudades y & incursionar y posicionarse en \\
& comunidades sostenibles (ODS 11); & mercados internacionales, que a su \\
& y Acción por el clima (ODS 13). & vez incrementan sus ganancias.
\end{tabular}

Nazzaro et 3 empresas Se analizaron las iniciativas de RSE

al. (2020) exportadoras del Sur mediante las dimensiones de Italia: 1) Molino de ambiental, económica y social, y aceite de oliva, 2) además implementaron un modelo Fábrica de pasta y 3) de ciclo de vida de RSE (Etapa Empresa de licores.
Inicial, de Desarrollo y de Madurez) para saber en qué etapa se encuentran las iniciativas de RSE de las empresas objeto de estudio. Los resultados evidenciaron que las tres empresas exportadoras si implementan iniciativas de RSE y que dichas iniciativas se encuentran todavía en una etapa inicial del ciclo de vida.
Se demostró que las prácticas de RSE pueden afectar los procesos comerciales, el proceso de producción y/o las características del producto. El molino de aceite de oliva y la fábrica de pasta han logrado reducir sus costos de producción y de transacción, además de generar menor impacto ambiental, que inciden positivamente en la creación de valor. Esto conlleva al aumento significativo de sus exportaciones.

$\begin{array}{ll}\text { Arredondo } & \text { Empresa exportadora } \\ \text { y Caldera } & \text { de calzado de México. } \\ (2021) & \end{array}$

(2021)
Los autores midieron la RSE de la empresa objeto de estudio en base a las 31 prácticas del World Economic Forum. Se evidenció que la empresa desarrolla acciones para hacer consciencia de que a mayor crecimiento mayor es el impacto que debe hacer a la sociedad, medio ambiente y economía.
La implementación de estas prácticas le ha permitido a la empresa la mejora en la optimización de sus procesos, ofrecer a sus clientes productos amigables con el medio ambiente y llegar a nuevos mercados tanto nacionales como internacionales.
Se analizaron las actividades de Medio ambiente.

$\begin{array}{llll}\text { Omazić } & \text { grandes empresas } & \text { RSE de las empresas exportadoras planifica } \\ \text { Aleksić } & \text { exportadoras } & \text { de } & \text { mediante las dimensiones de la ISO contribure } \\ (2021) & \text { Croacia. } & & 26000 \text { y se demostró que todas las compet } \\ & & \text { dimensiones son implementadas influencia } \\ & & \text { por dichas empresas pero las que significat } \\ & \text { más se destacan son: Prácticas de RSE } \\ & \text { laborales; Derechos humanos y la RSE. }\end{array}$

$\begin{array}{llll}\text { Omazić } & \text { grandes empresas } & \text { RSE de las empresas exportadoras planifica } \\ \text { Aleksić } & \text { exportadoras } & \text { de } & \text { mediante las dimensiones de la ISO contribure } \\ (2021) & \text { Croacia. } & & 26000 \text { y se demostró que todas las compet } \\ & & \text { dimensiones son implementadas influencia } \\ & & \text { por dichas empresas pero las que significat } \\ & \text { más se destacan son: Prácticas de RSE } \\ & \text { laborales; Derechos humanos y la RSE. }\end{array}$

$\begin{array}{llll}\text { Omazić } & \text { grandes empresas } & \text { RSE de las empresas exportadoras planifica } \\ \text { Aleksić } & \text { exportadoras } & \text { de } & \text { mediante las dimensiones de la ISO contribure } \\ (2021) & \text { Croacia. } & & 26000 \text { y se demostró que todas las compet } \\ & & \text { dimensiones son implementadas influencia } \\ & & \text { por dichas empresas pero las que significat } \\ & \text { más se destacan son: Prácticas de RSE } \\ & \text { laborales; Derechos humanos y la RSE. }\end{array}$

$\begin{array}{llll}\text { Omazić } & \text { grandes empresas } & \text { RSE de las empresas exportadoras planifica } \\ \text { Aleksić } & \text { exportadoras } & \text { de } & \text { mediante las dimensiones de la ISO contribure } \\ (2021) & \text { Croacia. } & & 26000 \text { y se demostró que todas las compet } \\ & & \text { dimensiones son implementadas influencia } \\ & & \text { por dichas empresas pero las que significat } \\ & \text { más se destacan son: Prácticas de RSE } \\ & \text { laborales; Derechos humanos y la RSE. }\end{array}$

$\begin{array}{llll}\text { Omazić } & \text { grandes empresas } & \text { RSE de las empresas exportadoras planifica } \\ \text { Aleksić } & \text { exportadoras } & \text { de } & \text { mediante las dimensiones de la ISO contribure } \\ (2021) & \text { Croacia. } & & 26000 \text { y se demostró que todas las compet } \\ & & \text { dimensiones son implementadas influencia } \\ & & \text { por dichas empresas pero las que significat } \\ & \text { más se destacan son: Prácticas de RSE } \\ & \text { laborales; Derechos humanos y la RSE. }\end{array}$

$\begin{array}{llll}\text { Omazić } & \text { grandes empresas } & \text { RSE de las empresas exportadoras planifica } \\ \text { Aleksić } & \text { exportadoras } & \text { de } & \text { mediante las dimensiones de la ISO contribure } \\ (2021) & \text { Croacia. } & & 26000 \text { y se demostró que todas las compet } \\ & & \text { dimensiones son implementadas influencia } \\ & & \text { por dichas empresas pero las que significat } \\ & \text { más se destacan son: Prácticas de RSE } \\ & \text { laborales; Derechos humanos y la RSE. }\end{array}$

$\begin{array}{llll}\text { Omazić } & \text { grandes empresas } & \text { RSE de las empresas exportadoras planifica } \\ \text { Aleksić } & \text { exportadoras } & \text { de } & \text { mediante las dimensiones de la ISO contribure } \\ (2021) & \text { Croacia. } & & 26000 \text { y se demostró que todas las compet } \\ & & \text { dimensiones son implementadas influencia } \\ & & \text { por dichas empresas pero las que significat } \\ & \text { más se destacan son: Prácticas de RSE } \\ & \text { laborales; Derechos humanos y la RSE. }\end{array}$

Se evidenció que si la RSE se planifica como una actividad que contribuye a lograr una ventaja competitiva puede tener una influencia estadísticamente significativa tanto en las actividades de RSE como en la comunicación de

$\begin{array}{llll}\text { Omazić } & \text { yrandes empresas } & \text { RSE de las empresas exportadoras planific } \\ \text { Aleksić } & \text { exportadoras } & \text { de } & \text { mediante las dimensiones de la ISO contribu } \\ (2021) & \text { Croacia. } & & 26000 \text { y se demostró que todas las compet } \\ & & \text { dimensiones son implementadas influencia } & \text { por dichas empresas pero las que significat } \\ & & \text { más se destacan son: Prácticas de RSE } \\ & & \text { laborales; Derechos humanos y la RSE. }\end{array}$

Fuente: Elaboración propia a partir de los autores citados.

De los estudios analizados, todos presentaron casos de empresas que desarrollan prácticas o acciones de RSE ligadas a las dimensiones social, económica y ambiental (Colovic y Henneron 2018; Martos et al. 2019; Nazzaro et al. 2020; Nyuur et al. 2019), a las dimensiones de la ISO 26000 (Barić et al. 2021; Granados et al. 2017) al World Economic Forum (Arredondo y Caldera, 2021), a los Objetivos de Desarrollo Sostenible (Mejía y Bom, 2020) y a los estándares de las certificaciones ambientales o de responsabilidad social (Moncada et al. 2019; Mora et al. 2019; Morán et al. 2019; Santa y Fajardo, 2017). 
Asimismo, de los estudios sistematizados se pudo constatar casos de empresas que además de implementar prácticas de RSE, se enfocaron también en los atributos del producto. Por ejemplo, Santa y Fajardo (2017) evidenciaron el caso de Café Quindío, que además de realizar prácticas sociales y medioambientales, certifica su café orgánico mediante la USDA Organic. Asimismo, Moncada et al. (2019) y Mora et al. (2019) evidenciaron casos de empresas exportadoras ecuatorianas que además de realizar prácticas sostenibles en base a certificaciones ambientales y de responsabilidad social, destacan los atributos de sus productos (camarón, banano, café, flores y cacao) mediante certificaciones orgánicas como: la USDA Organic, la Certificación francesa AB, entre otras.

Esto se puede explicar parafraseando a Barin et al. (2015) quienes argumentaron que las empresas exportadoras pueden complementar ambas estrategias de diferenciación basada en la RSE, en caso de que las prácticas o las características de RSE de los productos no sean suficientes para diferenciarse por sí solas. No obstante, ambas estrategias deben ser complementarias, por ejemplo, si las empresas exportadoras no lanzan productos con atributos sociales o ambientales, sus prácticas de RSE comienzan a perder valor.

En cuanto a las ventajas competitivas que obtuvieron las empresas exportadoras gracias las prácticas de RSE o la incorporación de características ambientales en sus productos, se puede decir que estas estrategias les han permitido a las empresas internacionalizarse, incursionar $\mathrm{y}$ consolidarse en mercados exigentes (con altas expectativas de RSE) donde además tienen la oportunidad de cobrar precios superiores. Asimismo, les han permitido generar confianza a sus consumidores internacionales lo que significa una imagen positiva y una buena reputación a favor. Y sobre todo, les han permitido diferenciarse de sus competidores y hacer que sus productos sean preferidos.

Estos resultados refuerzan las nociones teóricas de Lara y Sánchez (2021) y Godos et al. (2018) quienes han mencionado que la RSE aumenta las posibilidades de que una empresa se internacionalice. Asimismo, se refuerza lo mencionado por Boehe y Barin (2010) quienes sostuvieron que los clientes internacionales ven a la RSE como algo superior y compensarán aquello pagando precios superiores. A esto se suman las ideas de Barić y Omazić (2018), Barić, Omazić y Aleksić (2019), Godos et al. (2018), Lu et al. (2020) y Vu et al. (2020), quienes argumentaron que la RSE permite a las empresas generar confianza a sus partes interesadas, crear una imagen y reputación positiva por lo que las posibilidades de que prefieran sus productos aumentan.

Por otro lado, considerando que "el grado de diferenciación [...] está determinado en última instancia por las percepciones de los consumidores" (Li, Zhang y Sun, 2019, p. 2) es necesario entonces que estos tengan conocimiento sobre las prácticas o atributos de RSE implementadas por las empresas exportadoras para que actúen en forma de compensación (Maldonado, Pinzón y Leana, 2017). Es por ello que además de un comportamiento socialmente responsable, las empresas deben desarrollar estrategias de comunicación dirigidas a sus partes interesadas ( $\mathrm{Lu}$, Ren, He, Lin y Streimikis, 2019) principalmente a sus consumidores (Li et al. 2019). 
De hecho, de los 12 estudios considerados, tres de ellos incluyeron en sus análisis la comunicación. Por ejemplo, Mejía y Bom (2020) en su estudio mencionaron que, para Cementos Argos S.A., evidenciar sus acciones de RSE a sus clientes actuales y potenciales mediante informe de sostenibilidad es un factor clave que le ha permitido ser un referente internacional. Por otra parte, Barić et al. (2021) mediante un análisis empírico lograron evidenciar que las 78 medianas y grandes empresas exportadoras de Croacia comunican sus actividades de RSE mediante informes de RSE, páginas web, redes sociales y publicidad, siendo los informes de RSE y las páginas web los principales. Finalmente, Martos et al. (2019) recomiendan a los gerentes de las empresas exportadoras del sector agroalimentario de Almería, desarrollar una estrategia de comunicación enfocada en cuestiones sociales y medioambientales, siendo las certificaciones las ideales para este propósito.

Tomando en cuenta la recomendación brindada por Martos et al. (2019) se puede decir que Café Quindío de Colombia (Santa y Fajardo, 2017), las cinco empresas camaroneras ecuatorianas (Moncada et al. 2019), las 15 exportadoras ecuatorianas del sector cacao, banano, camarón, café y flores (Mora et al. 2019) y la Hacienda bananera Nueva Colonia de Ecuador (Morán et al. 2019) comunican sus prácticas y/o atributos de RSE mediante sus certificaciones.

En síntesis, los canales de comunicación más usados por las empresas exportadoras para dar a conocer a sus clientes sus estrategias de diferenciación basada en la RSE, son las certificaciones, los informes de RSE y las páginas web.

\section{Conclusiones}

Desde el marco de la teoría instrumental, la RSE se posiciona como un enfoque estratégico que contribuye a generar ventajas competitivas. Así la adopción de la RSE es útil para las empresas que se enfrentan a las necesidades de un grupo de consumidores que se preocupan por la relación de los productos que consumen y las acciones de las empresas que los proveen en el medio ambiente y la sociedad.

En función del objetivo planteado, se concluye que las empresas exportadoras implementan estrategias de diferenciación mediante la aplicación de protocolos de RSE tanto a nivel producto como a nivel empresa, siendo esta última la de mayor uso. Esta dualidad es necesaria para cubrir las necesidades de mercados exigentes. No se podría considerar socialmente responsable a las empresas que por un lado realizan prácticas de RSE mientras que sus productos o sus procesos productivos tienen consecuencias negativas para la comunidad o el medio ambiente.

Es importante destacar de los hallazgos obtenidos que la comunicación de las prácticas de RSE es un factor fundamental para que las empresas exportadoras extiendan su reputación y hagan visible sus compromisos. De acuerdo a los estudios analizados, las certificaciones, los informes de RSE y las páginas web son los medios que utilizan las empresas para difundir su aplicación a los grupos de interés. Las certificaciones son las más usadas para este fin por el mayor nivel de confiabilidad que brinda a los consumidores. Tal como lo explica Barin et al. (2015) los informes de RSE son realizados por las propias empresas, mientras que las certificaciones son emitidas y 
monitoreadas por entidades certificadoras independientes. En cuanto a la página web que ocupa el tercer lugar, actúan como un canal de comunicación ya que allí las empresas crean una sección específica relacionada a la RSE o a la sustentabilidad para publicar sus informes anuales, así como sus certificaciones y reconocimientos obtenidos en esta área (Bogetić et al. 2018).

Este estudio tiene implicaciones para la gestión de las empresas exportadoras, coincidiendo con varios autores al sustentar que la adopción de RSE identificada como estrategia de diferenciación contribuye a mejorar la posición competitiva en los mercados internacionales. Lo expuesto se verifica en la existencia de un creciente grupo de importadores de segmentos de mercados especializados (preocupados por la responsabilidad social y ambiental), que en Estados Unidos y Europa buscan hacer negocios con empresas exportadoras que puedan respaldar que sus productos proceden de programas de producción en el marco de las certificaciones de RSE. Los importadores se están sumando a distintas iniciativas de producción socialmente responsables, incluso con sus propios códigos de conducta, lo que va fortaleciendo una demanda especializada a la que deben responder las empresas.

Finalmente, se recomienda tener en cuenta las limitaciones de este estudio, al tratarse de una investigación documental, con enfoque cualitativo y alcance descriptivo, se emplearon los estudios precedentes de otros autores que analizaron la situación de empresas exportadoras de diferentes tamaños, sectores productivos y países, por lo que los resultados no podrían ser generalizados. No obstante, las futuras investigaciones podrían realizar estudios estadísticos correlacionales para identificar el tipo de relación entre la variable estrategia de diferenciación (basada en la RSE) con la variable ventaja competitiva. Así también, se podría considerar medir la relación entre ambas variables teniendo como variable mediadora los medios de comunicación de la RSE.

\section{Referencias bibliográficas}

Arredondo-Hidalgo, M. G. y Caldera González, D. C. (2021). Análisis de la RSE y el impacto de la cadena de suministro en las prácticas de desempeño sustentable: un estudio de caso en una empresa exportadora mexicana. Investigación \& Desarrollo, 29(1), 15-40. https://bit.ly/2TG6UQR

Barić, A. y Omazić, M. A. (2018). Role of Corporate Social Responsibility in Croatian Export Companies. Proceedings of the ENTRENOVA - Enterprise Research Innovation Conference, 4(1), 408-416. https://hrcak.srce.hr/ojs/index.php/entrenova/article/view/13943

Barić, A., Omazić, M. A. y Aleksić, A. (2019). Corporate Social Responsibility and Stakeholders' Management of Export Companies in Foreign Markets. Proceedings of the ENTRENOVA - Enterprise Research Innovation Conference, 5(1), 128-135. https://hrcak.srce.hr/ojs/index.php/entrenova/article/view/13750

Barić, A., Omazić, M. A. y Aleksić, A. (2021). Corporate social responsibility of export organizations: relation between strategy, activities and communication on foreign markets. Interdisciplinary Description of Complex Systems, 19(1), 120-131. https://doi.org/10.7906/indecs.19.1.10

Barin Cruz, L., Boehe, D. M. y Ogasavara, M. H. (2015). CSR-based Differentiation Strategy of Export Firms from Developing Countries: An Exploratory Study of the Strategy Tripod. Business \& Society, 54(6), 723 -762. https://doi.org/10.1177/0007650312473728 
Boehe, D. M. y Barin Cruz, L. (2010). Corporate Social Responsibility, Product Differentiation Strategy and Export Performance. Journal of Business Ethics, 91, 325-346. https://doi.org/10.1007/s10551-010-0613-z

Bogetić, S., Đorđević, D., Ćoćkalo, D. y Vorkapić, M. (2018). Corporate social responsibility as a factor of global competitiveness. Journal of Engineering Management and Competitiveness (JEMC), 8(1), 11-19. https://scindeks.ceon.rs/article.aspx?artid=233496381801011B

Boubakary y Moskolaï, D. D. (2016). The influence of the implementation of CSR on business strategy: An empirical approach based on Cameroonian enterprises. Arab Economic and Business Journal, 11(2), 162-171. https://doi.org/10.1016/j.aebj.2016.04.001

Carroll, A. B. (1979). A Three-Dimensional Conceptual Model of Corporate Performance. Academy of Management Review, 4(4), 497-505. https://doi.org/10.5465/amr.1979.4498296

Carroll, A. B. (2008). A History of Corporate Social Responsibility: Concepts and Practices. En A. Crane, D. Matten, A. McWilliams, J. Moon y D. S. Siegel. (Eds). The Oxford Handbook of Corporate Social Responsibility. (pp. 19-46). Oxford University Press. http://doi.org/10.1093/oxfordhb/9780199211593.003.0002

Carroll, A. B. y Brown, J. A. (2018). Corporate Social Responsibility: A Review of Current Concepts, Research and Issues. En J. Weber y D.M. Wasieleski. (Eds.) Corporate Social Responsibility. (pp. 39-69). U.K.: Emerald Publishing Co.

Castejón, P. J. M. y López, B. A. (2016). Corporate social responsibility in family SMEs: A comparative study. European Journal of Family Business, 6(1), 21-31. https://doi.org/10.1016/j.ejfb.2016.05.002

Castro Alfaro, A. (2017). La responsabilidad social empresarial y la competitividad en las organizaciones empresariales. Revista Innova ITFIP, 1(1), 78-89. http://www.revistainnovaitfip.com/index.php/innovajournal/article/view/16

Chavarría Ortiz, C., Ganga Contreras, F. y García Parejo, A. (2020). Responsabilidad Social Corporativa (RSC): impacto en pequeñas y medianas empresas españolas. Revista Venezolana de Gerencia (RVG), 25(91), 1056-1075. https://doi.org/10.37960/rvg.v25i91.33182

Colovic, A. y Henneron, S. (2018). Corporate Social Responsibility and SME Internationalization: Insights from the Food Industry. Management international / International Management / Gestiòn Internacional, 22, 30-41. https://doi.org/10.7202/1062492ar

Cueto Cedillo, C. y de la Cuesta González, M. (2019). La administración pública de la responsabilidad social corporativa (2. ${ }^{\mathrm{a}}$ ed.). Editorial Área de Innovación y Desarrollo,S.L. http://doi.org/10.17993/EcoOrgyCso.2019.53

Daniel Licandro, O., Alvarado-Peña, L. J., Sansores Guerrero, E. A. y Navarrete Marneou, J. E. (2019). Responsabilidad Social Empresaria: Hacia la conformación de una tipología de definiciones. Revista Venezolana De Gerencia, 24(85), 281-299. https://doi.org/10.37960/revista.v24i85.23843

Flores-Ortiz, M. V., Vega-López, A. y Chávez-Moreno, E. A. (2016). La responsabilidad social empresarial como factor de competitividad en la Industria maquiladora de Tijuana, B.C., México. Revista Internacional Administración \& Finanzas, 9(4), 65-77. https://bit.ly/2VjRKRD 
Fong, C., Parra, A., Soriano, L. y Teodoro, E. (2020). El estado actual de la Responsabilidad Social Empresarial en México. Revista Academia \& Negocios, 6(1), 41-56. https://revistas.udec.cl/index.php/ran/article/view/2618

Garriga, E. y Melé, D. (2004). Corporate Social Responsibility Theories: Mapping the Territory. Journal of Business Ethics, 51-71. https://doi.org/10.1023/B:BUSI.0000039399.90587.34

Godos-Díez, J. L., Cabeza-García, L. y Fernández-González, C. (2018). Relación entre la Responsabilidad Social Corporativa (RSE) y las estrategias de Internacionalización: un estudio descriptivo en el contexto español. Ciencias Administrativas, 8(4), 57. https://doi.org/10.3390/admsci8040057

Maldonado-Guzman, G., Pinzón-Castro S. Y. y Leana-Morales, C. (2017). Corporate Social Responsibility, Brand Image and Firm Reputation in Mexican Small Business. Journal of Management and Sustainability, 7(3), 38-47. http://doi.org/10.5539/jms.v7n3p38

Granados Mendoza, J. A., Arredondo Hidalgo, M. G. y Caldera González, D. C. (2017). Las pymes exportadoras de Guanajuato y su visión de la responsabilidad social empresarial RSE. Jóvenes en la ciencia, 3(2), 433-455. https://bit.ly/316BuhV

Kim, S. (2019). The Process Model of Corporate Social Responsibility (CSR) Communication: CSR Communication and its Relationship with Consumers' CSR Knowledge, Trust, and Corporate Reputation Perception. Journal of business ethics, 154, 1143-1159. https://doi.org/10.1007/s10551-017-3433-6

Kolk, A. (2016). The social responsibility of international business: From ethics and the environment to CSR and sustainable development. Journal of World Business, 51(1), 2334. https://doi.org/10.1016/j.jwb.2015.08.010

Lara Manjarrez, I. A. y Sánchez Gutiérrez, J (2021). Responsabilidad social empresarial para la competitividad de las organizaciones en México. Mercados y Negocios, 2(43), 97-108. https://doi.org/10.32870/myn.v0i43.7546

Latapí Agudelo, M. A., Jóhannsdóttir, L. y Davídsdóttir, B. (2019). A literature review of the history and evolution of corporate social responsibility. International Journal of Corporate Social Responsibility, 4(1). https://doi.org/10.1186/s40991-018-0039-y

Li, J., Zhang, F. y Sun, S. (2019). Building Consumer-Oriented CSR Differentiation Strategy. Sustainability, 11(3), 664. https://doi.org/10.3390/su11030664

Lizcano-Prada, J. y Lombana, J. (2018). Responsabilidad Social Corporativa (RSC): reconsiderando conceptos y enfoques. Revista Civilizar, 18(34), 119-134. https://doi.org/10.22518/usergioa/jour/ccsh/2018.1/a08

Lu, J., Ren, L., He, Y., Lin, W. y Streimikis, J. (2019). Linking Corporate Social Responsibility with Reputation and Brand of the Firm. Amfiteatru Economic, 21(51), 442-460. https://www.amfiteatrueconomic.ro/ArticolEN.aspx?CodArticol=2824

Lu, J., Ren, L., Yao, S., Qiao, J., Mikalauskiene, A. y Streimikis, J. (2020). Exploring the relationship between corporate social responsibility and firm competitiveness. Economic Research-Ekonomska Istraživanja, $\quad 33(1), \quad 1621-1646$. https://doi.org/10.1080/1331677X.2020.1761419

Marakova, V., Wolak-Tuzimek, A. y Tuckova, Z. (2021). Corporate Social Responsibility as a Source of Competitive Advantage in Large Enterprises. Journal of Competitiveness, 13(1), 113-128. https://doi.org/10.7441/joc.2021.01.07 
Martos-Pedrero, A., Cortés-García, F. J. y Jiménez-Castillo, D. (2019). The Relationship between Social Responsibility and Business Performance: An Analysis of the Agri-Food Sector of Southeast Spain. Sustainability, 11(22), 6390. https://doi.org/10.3390/su11226390

Mateos Espejel, L., Estrada Rodríguez, J. L. y Hernández Ruiz, H. M. (2020). Competitividad y responsabilidad social desde la comunicación de la ciencia. Revista Venezolana De Gerencia, 25(91), 995-1013. https://doi.org/10.37960/rvg.v25i91.33178

Mejía-Bermúdez, A. y Bom-Camargo, Y. I. (2020). Responsabilidad social empresarial para la apertura de mercados internacionales. Revista Venezolana De Gerencia, 24(2), 251-269. https://doi.org/10.37960/revista.v24i2.31492

Moncada-Sánchez, G. G., Ramírez-Quevedo, P. X. y González-Illescas, M. L. (2019). Estrategias competitivas de las empresas ecuatorianas exportadoras de camarón. Casos de éxito. $\begin{array}{llll}\text { INNOVA Research } & \text { Journal, } & \text { 125-142. }\end{array}$ https://doi.org/10.33890/innova.v5.n1.2020.1115

Mora-Córdova, D. E., Lituma-Loja, A. A. y González-Illescas, M. L. (2019). Las certificaciones como estrategia para la competitividad de las empresas exportadoras. INNOVA Research Journal, 5(2), 113-132. https://doi.org/10.33890/innova.v5.n2.2020.1274

Morán Montalvo, C. O., Cárdenas Zambrano, C. S. y Córdova Serrano, C. E. (2019). Características de la responsabilidad social empresarial de las Pymes en Ecuador. Caso de estudio: Hacienda Nueva Colonia. Revista ECA Sinergia, 10(3) 131-144. https://doi.org/10.33936/eca_sinergia.v10i3.1558

Nazzaro, C., Stanco, M. y Marotta, G. (2020). The Life Cycle of Corporate Social Responsibility in Agri-Food: Value Creation Models. Sustainability, 12(4), 1287. https://doi.org/10.3390/su12041287

Nyuur, R. B., Ofori, D. F. y Amponsah, M. M. (2019). Corporate social responsibility and competitive advantage: A developing country perspective. Thunderbird International Business Review, 61, 551- 564. https://doi.org/10.1002/tie.22065

Ormaza Andrade, J., Ochoa-Crespo, J., Ramírez Valarezo, F. y Quevedo Vázquez, J. (2020). Responsabilidad social empresarial en el Ecuador: Abordaje desde la Agenda 2030. Revista De Ciencias Sociales, 26(3), 175-193. https://doi.org/10.31876/rcs.v26i3.33241

Parrales Zumba, C. G., Trelles Vera, G. M. y González Illescas, M. L. (2021). La responsabilidad social empresarial y su papel estratégico en la competitividad de las empresas exportadoras $\begin{array}{llll}\text { internacionales. Yachana Revista } \quad \text { Científica, } & \text { 10(2), }\end{array}$ http://revistas.ulvr.edu.ec/index.php/yachana/article/view/670

Porter, M. E. (1985). Competitive Advantage. The Free Press.

Porter, M. E. y Kramer, M. R. (2006). Strategy and society: The link between competitive advantage and corporate social responsibility. Harvard Business Review, 84(12), 78-92. https://bit.1y/3x702rD

Rueda Galvis, J. F. (2017). La responsabilidad social empresarial como estrategia que contribuye al éxito corporativo. En J. A. Cortés y E. Flores Córdova. (Eds.). Responsabilidad social (pp. 10-26). Centro de Investigación y Desarrollo Ecuador. https://bit.ly/3i8kbuU

Santa Fajardo, L. M. y Fajardo Calderón, C. L. (2017). La responsabilidad social empresarial (RSE), estrategia efectiva para la internacionalización y competitividad de las empresas del Quindío. En J. A. Cortés y E. Flores Córdova. (Eds.). Responsabilidad social (pp. 27-42). Centro de Investigación y Desarrollo Ecuador. https://bit.ly/3i8kbuU 
Suárez Rodríguez, O. y Yance Acurio, H. (2020). Diagnóstico de la responsabilidad social empresarial en las pymes de la ciudad de Guayaquil. Revista Mapa, 4(18), 169-184. http://revistamapa.org/index.php/es/article/view/233

Tan, K. L., Ho, J. M., Pidani, R. y Goveravaram, A. D. (2021). Doing good does you good? The financial impact of individual CSR dimensions: A Malaysian context. Social Responsibility Journal. https://doi.org/10.1108/SRJ-04-2020-0146

Villafán-Vidales, K. B. (2020). Evolución conceptual de la responsabilidad social. Revista de la Facultad de Contaduría y Ciencias Administrativas, 5(10), 69-80. https://rfcca.umich.mx/index.php/rfcca/article/view/145

Vu, T. L, Nguyen, N. T., Nguyen, X. H., Nguyen, Q. H. y Nguyen, H. L. (2020). Corporate social responsibility, employee commitment, reputation, government support and financial performance in Vietnam's export textile enterprises. Accounting, 6(6), 1045-1058. http://dx.doi.org/10.5267/j.ac.2020.7.015 